\title{
Obstetrical outcome in the third trimester after hysteroscopic adhesiolysis
}

\author{
Qing Feng ${ }^{1}$, Bingsi Gao ${ }^{1}$, Huan Huang ${ }^{1}$, Jeffrey Je-Chuen Woo ${ }^{2}$, Lingxiao Zou ${ }^{1}$, Xingping Zhao ${ }^{1}$, \\ Chunxia Cheng', Dabao Xu ${ }^{1}$ \\ ${ }^{1}$ Department of Obstetrics and Gynecology, The Third Xiangya Hospital of Central South University, Changsha 410013, China; ${ }^{2}$ Department of \\ Obstetrics and Gynecology, Riverside Regional Medical Center, Newport News, VA, USA \\ Contributions: (I) Conception and design: D Xu, C Cheng; (II) Administrative support: D Xu; (III) Provision of study materials or patients: Q Feng, \\ X Zhao; (IV) Collection and assembly of data: Q Feng, B Gao, JJ Woo; (V) Data analysis and interpretation: Q Feng, B Gao, H Huang, L Zou; (VI) \\ Manuscript writing: All authors; (VII) Final approval of manuscript: All authors. \\ Correspondence to: Dabao Xu, MD; Chunxia Cheng, MD. Department of Obstetrics and Gynecology, The Third Xiangya Hospital of Central South \\ University, Changsha 410013, China. Email: dabaoxu@yahoo.com; darlingch@126.com.
}

\begin{abstract}
Background: Intrauterine adhesion (IUA) is caused by adhesion of the uterine cavity due to the damage of endometrium. Hysteroscopic adhesiolysis (HA) is the main treatment. The objective of the study was to investigate the obstetrical outcome in the third trimester of women who previously underwent HA.

Methods: We performed a retrospective cohort study in the university-affiliated hospital. A total of 146 women with a history of HA who had given birth in their third trimester from May 2012 to May 2019 were enrolled (study group), while 292 women with a negative history of HA were matched for maternal age, gravidity, parity, and delivery year. The parameters of obstetrics and infants were investigated to evaluate the change in the third trimester of women with a history of HA.

Results: There was no significant difference between study and control groups in gestational weeks, nor in fetal gender distribution, birth weight, Apgar score, fetal distress, and neonatal intensive care unit (NICU) admissions after delivery $(\mathrm{P}>0.05)$. In addition, when compared with the control group, women with a history of HA were at a higher risk of placental risks $(\mathrm{P}<0.05)$, such as placenta previa $(11.6 \%$ versus $3.1 \%)$, abnormally invasive placenta (AIP) (33.56\% versus $2.7 \%$ ), and retained placenta (42.5\% versus $8.6 \%$ ). This resulted in a significantly higher postpartum hemorrhage $(\mathrm{PPH})$ rate in the study group as compared with that in the control group $(8.9 \%$ versus $1.0 \%, \mathrm{P}<0.05)$. Such cases were more likely to be found in patients with severe IUA compared with those who were assessed as mild and moderate.
\end{abstract}

Conclusions: The history of HA might be an important risk factor inducing placental problems and $\mathrm{PPH}$ in the third trimester. More attention should be paid to the labor of pregnant women with a history of HA.

Keywords: Asherman syndrome; hysteroscopy; pregnancy; obstetrical outcome; placenta

Submitted Sep 08, 2019. Accepted for publication Sep 18, 2019.

doi: 10.21037/atm.2019.09.123

View this article at: http://dx.doi.org/10.21037/atm.2019.09.123

\section{Introduction}

Intrauterine adhesion (IUA), also known as Asherman syndrome, is caused by adhesion of the uterine cavity due to the damage of endometrium. Its factors include surgical abortion, cesarean section, uterine myomectomy, hysteroscopic resection of endometrial polyps (HREP), intrauterine contraceptive device (IUD) placing or delivery, endometrial tuberculosis, uterine artery ligation or embolization, and pelvic radiotherapy $(1,2)$. Patients with IUA generally present with hypomenorrhea or amenorrhea, recurrent pregnancy loss, and infertility. IUA patients may have a low pregnancy rate in receiving assisted reproductive techniques (ARTs). During the last two 
decades, the advent of hysteroscopy has revolutionized the diagnosis and management of IUA $(3,4)$. The latest reports have recorded the chance of pregnancy after transcervical resection of adhesion (TCRA) was $79.0 \%$ and the chance of a live birth was $63.7 \%$ (5). Several studies have been carried out to evaluate the reproductive outcome in cases after hysteroscopic adhesiolysis (HA) (6). Although HA is considered reasonably safe, its effects on obstetrical outcomes remain uncertain. Previous studies with small case numbers have focused on pregnancy data in patients with HA, including pregnancy rates, abortion rates, and gestational time $(3,7)$.

The present retrospective study analyzed the third trimester obstetrical outcomes in 146 pregnant women with a history of HA (study group) and 292 pregnant women without a history of HA (control group). The primary obstetrical outcomes including cesarean-section rate, placental problems [i.e., placenta praevia, abnormally invasive placenta (AIP), and retained placenta] and postpartum hemorrhage (PPH) of the study and control group were compared.

\section{Methods}

This study was carried out at the Third Xiangya Hospital of Central South University. This matched cohort study was approved by the regional ethics committee in Hunan province, China (No. 2019-S242). We had access to 21,098 deliveries recorded in the third trimester in our hospital between May 2012 and May 2019, among whom 171 had a history of HA. We excluded mothers with multiple births (10/171, 5.85\%), intrauterine fetal death $(5 / 171,2.92 \%)$, operative vaginal delivery $(3 / 171$, $1.75 \%$ ), all of which may have altered the obstetrical outcomes. Additionally, 7 cases with incomplete medical records $(7 / 171,4.09 \%)$ were also excluded. Ultimately, 146 eligible cases on singleton live term in the third trimester were included in the study group. In addition, of the 20,927 pregnant women without a history of HA, 4,509 recorded progestational menstruation and received ultrasound imaging examination before pregnancy. Among them, 4,413 cases had no reduction in menstrual flow before pregnancy, with ultrasound image showing normal endometrium. The control group $(\mathrm{N}=292)$ was selected from 4,413 cases with the same exclusion criteria and matched on a 1:2 ratio with the study group; matching was performed by maternal age ( \pm 1 year), gravidity $( \pm 1)$, parity (primiparous or multiparous, \pm 1 ), and delivery year (2012-
$2019, \pm 1$ year) (Figure 1).

The IUA classification was evaluated according to the American Fertility Society (AFS) in 1988 (8). IUAs were classified into mild, moderate, and severe degree by scoring the extent of endometrial cavity obliteration, type of adhesions, and menstrual pattern, based on hysteroscopic surgery. All cases of IUA were diagnosed hysteroscopically and classified according to the classification based on the AFS by two experienced surgeons.

We reviewed basic information regarding each case, including maternal age, gravidity, parity, and gestational weeks, etc. Information regarding HA history were also collected, including adhesion scores pre-HA and the time interval between HA and natural pregnancy. Moreover, fetal outcomes of the two groups were collected, including fetal gender, weight, fetal distress, 1- and 5-minute Apgar score, and neonatal intensive care unit (NICU) admission. The main outcomes of the mother included cesarean-section rate, placental problems (i.e., placenta praevia, AIP and retained placenta) and $\mathrm{PPH}$ (defined as blood loss $>1,000$ $\mathrm{mL}$ within 24 hours following delivery) (9). AIP, also known as morbidly adherent placenta, is a broad term that describes abnormal adherence of placenta to the underlying myometrium. Depending on the depth of invasion, it is further defined as placenta accreta, placenta increta, and placenta percreta (10). The diagnosis of AIP was made based on findings obtained by color Doppler mapping, MRI, and pathological diagnosis of the placenta. Data acquisition and sorting were performed using Microsoft Excel 2013.

All statistical analyses were performed using SPSS Statistics 20 (IBM, SPSS, Chicago, IL, USA). Descriptive parameters are expressed as mean SD or median (range). In the matched study, continuous variables were compared using the mixed model for parametric variables, while the frequencies of categorical variables were compared using the conditional logistic regression model. First, correlation between interest factors and obstetrical outcome were assessed by the univariate analyses. Then, the significant variables were included in the multivariate logistic regressions to calculate $\mathrm{B}$ coefficient and standard error. A value of $\mathrm{P}<0.05$ was considered as significant difference.

\section{Results}

\section{General information}

A total of 146 pregnant women with a history of HA and the matched 292 pregnant women without a history of HA 


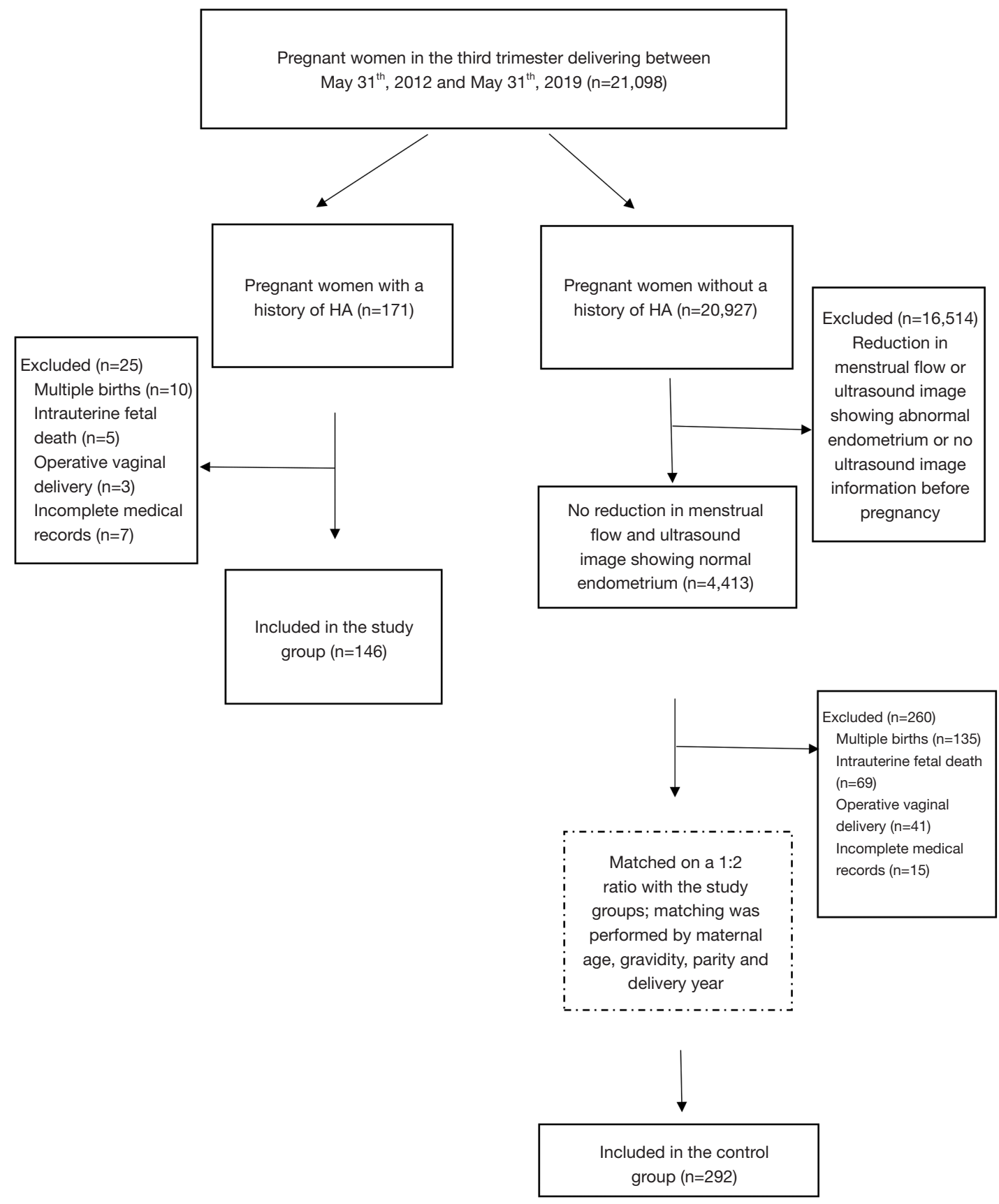

Figure 1 Flowchart of the study population.

were included in this study. The average age of pregnant women in the two groups was 31.2 years. The median quantities of gravidity of the two groups was 4, and the median quantities of the parity was 1 (Table 1). The average number of gestational weeks in the study and control groups were $38.6 \pm 2.29$ and $38.9 \pm 1.58$ respectively, the difference of which was not statistically significant $(\mathrm{P}>0.05)$.
The characteristics of both two groups are listed in Table 2.

\section{Obstetrical outcomes in two groups}

In the study group, 104 pregnant women (104/146, 71.2\%) had cesarean sections, corresponding to 132 pregnant women $(132 / 292,45.2 \%)$ in the control group. The 
Table 1 General information

\begin{tabular}{lccc}
\hline Features & $\begin{array}{c}\text { Delivery after } \\
\mathrm{HA}(\mathrm{n}=146)\end{array}$ & $\begin{array}{c}\text { Delivery without } \\
\text { receiving HA } \\
(\mathrm{n}=292)\end{array}$ & $\mathrm{P}$ value \\
\hline Maternal age years & $31.19 \pm 4.11$ & $31.19 \pm 4.12$ & 0.974 \\
Gravidity & $4.00(2.00,4.00)$ & $4.00(2.00,4.00)$ & 0.853 \\
Parity & $1.00(1.00,2.00)$ & $1.00(1.00,2.00)$ & 0.858 \\
\hline
\end{tabular}

Data are expressed as mean SD, not \%. P>0.05, not significant. HA, hysteroscopic adhesiolysis.

distribution had a significant statistical significance (OR $1.58 ; 95 \%$ CI, 1.22-2.04). After placenta delivery, the integrity of the placenta was checked by an experienced doctor. It was found that the placental integrity rate of the puerperae with the history of HA was significantly lower than that of the control group (OR 0.70; 95\% CI, $0.54-0.89)$. Placenta previa (11.6\%) was more likely to be implicated in the study group, which was higher than that of the control group (3.1\%), suggesting statistically significant distribution (OR 3.78; 95\% CI, 1.68-8.47). Sixty-two puerperae $(42.5 \%)$ had retained placenta in the study group, corresponding to $25(8.6 \%)$ in the control group, the difference of which was statistically significant (OR 5.00; 95\% CI, 3.12-7.89). Pregnant women with retained placenta were subjected to manual removal of the placenta after the umbilical vein was injected with oxytocin and the placenta failed to be delivered. In the study group, 49 patients suffered from AIP, including 26 cases of placenta accreta, 21 cases of placenta increta, and 2 cases of placenta percreta. While in the control group, there were 8 cases of AIP patients, including 6 cases of placenta accreta and 2 cases of placenta increta, with no cases of placenta percreta found. The distribution displayed a significant statistical significance (OR 17.93; 95\% CI, 8.18-39.33). The median quantities of postpartum blood loss within 24 hours in the study group was 455.0 (357.5, 630.0) $\mathrm{mL}$, which was significantly higher than $362.5(290.0,460.0) \mathrm{mL}$ in the control group $(\mathrm{P}<0.0001)$. The $\mathrm{PPH}$ rate $(8.9 \%)$ in the study group was significantly higher than that of the control group (1.0\%) (OR 9.33; $95 \%$ CI, 2.68-32.48). In the study group, 13 puerperae had PPH (range, 1,030-6,080 mL), of which 12 had AIP. One case in 13 patients received temporal internal iliac occlusion balloon catheters during cesarean section, 3 cases received uterine artery embolization, 4 cases used the Bakri balloon, and 2 cases were subjected to B-Lynch brace suture, while no hysterectomies were performed. However, in control group, 3 patients suffered $\mathrm{PPH}$, of whom, 1 received the Bakri balloon alone, 1 was subject to the B-Lynch brace suture, and the rest were simply treated with medication. Overall, $14.4 \%$ of puerperae needed blood transfusion, which was a rate significantly higher than that of the control group (0.3\%) (OR 42.00; 95\% CI, 5.65-312.2) (Table 2).

In addition, neonatal birth outcomes between the study group and control group were compared. There was no statistical significance difference in fetal gender distribution, gestational weeks, birth weight, Apgar score of 1 and 5 minutes after birth, fetal distress, and NICU admissions after childbirth between the study group and the control group $(\mathrm{P}>0.05)$ (Table 2).

\section{The relation between severity of IUA and the obstetrical outcomes}

The pregnant women with a history of HA were divided according to the severity degree of IUA. Because there were few cases where mild IUA caused infertility or a significant decrease in menstrual flow, the number of patients in the mild group was very small. Therefore, those with adhesion scored 1-8 were classified as the mild to moderate group $(\mathrm{n}=104)$, and those who scored 9-12 were classified as the severe group $(n=42)$. With logistic regression, the frequencies of categorical variables were maintained when adjusting for covariates of maternal age, gravidity, and parity.

In the severe group, cesarean-section rate, presence of placenta previa, and the time of becoming pregnant after HA, were not significantly different from those in the mild to moderate group $(\mathrm{P}>0.05)$. The average number of gestational weeks in the severe group and the mild-tomoderate group were $38.9 \pm 1.88$ and $37.83 \pm 2.98$ respectively, the difference of which was statistically significant $(\mathrm{P}<0.05)$. In the severe group, $57.1 \%$ of puerperae suffered from AIP, which was significantly higher than the percentage in the mild-to-moderate group (24\%) [adjusted risk ratio (aOR) 4.63; 95\% CI, 2.10-10.18]. Eleven patients were diagnosed with $\mathrm{PPH}$ (26.2\%) in the severe group, which was significantly higher than the 2 in the mild-to-moderate group (1.9\%) (OR 11.944; 95\% CI, 3.091-46.15), while blood transfusion in the severe group was significantly higher than that in the mild-to-moderate group (OR 9.193; 95\% CI, 3.173-26.63) (Table 3).

The neonatal birth outcomes between the severe group and the mild-to-moderate group were also compared. 
Table 2 The third trimester obstetrical outcomes among pregnant women in the study group and the control group

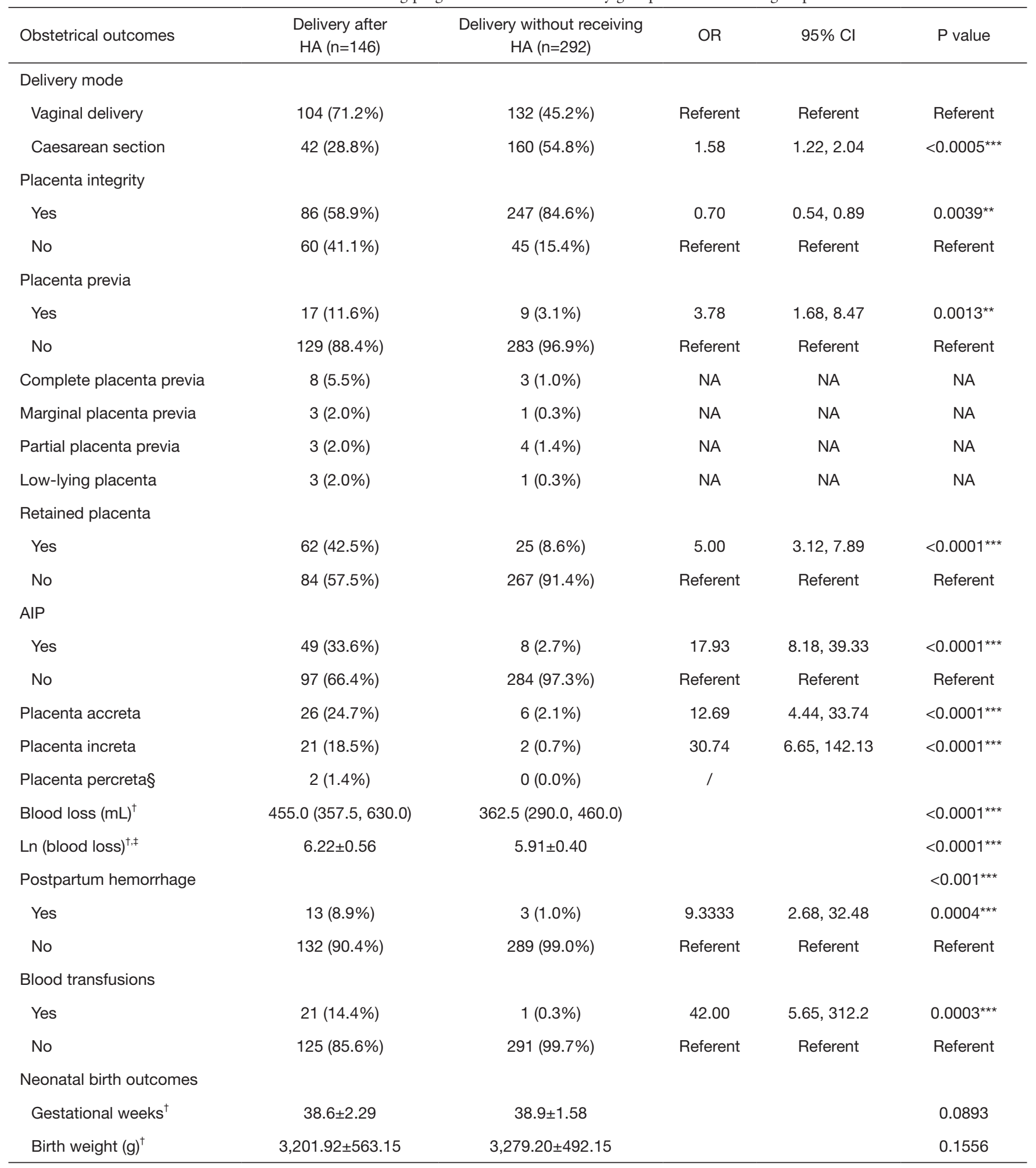

Table 2 (continued) 
Table 2 (continued)

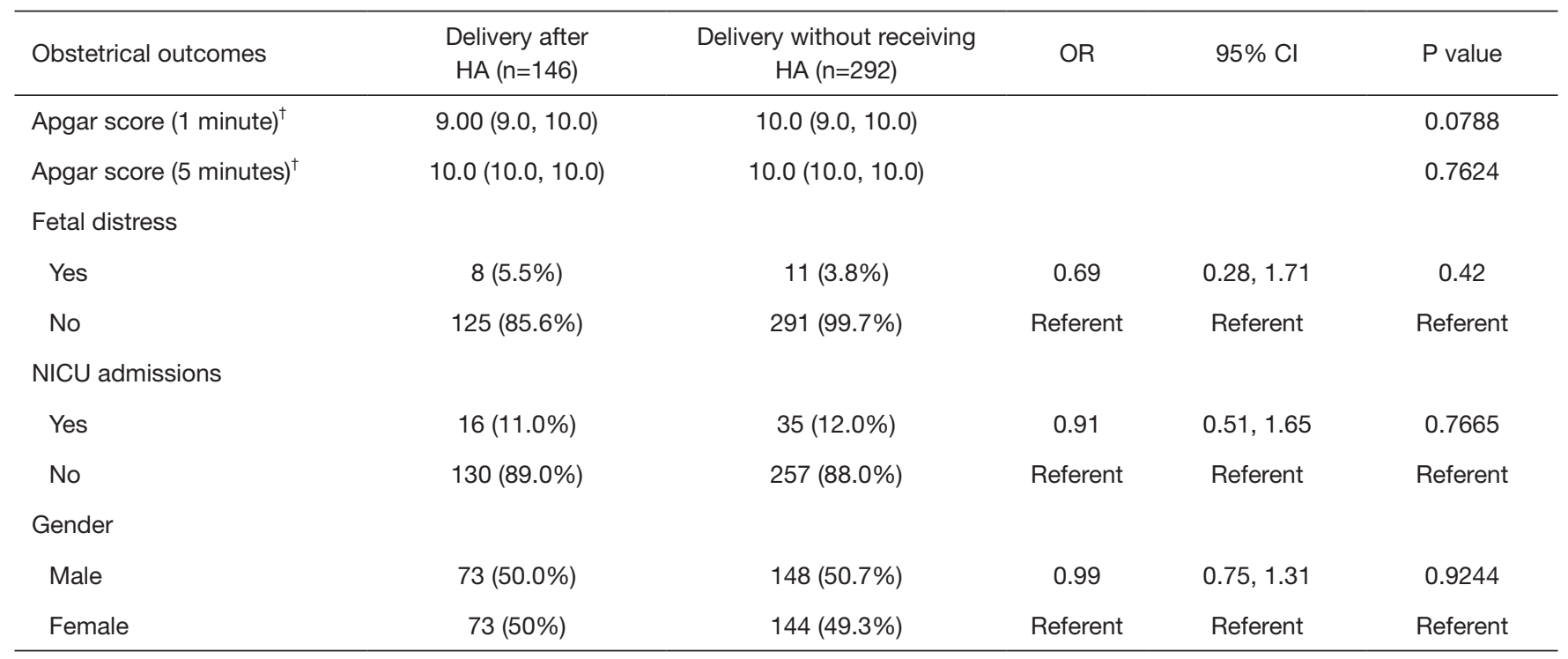

${ }^{\dagger}$, continuous variables were compared using the mixed model for parametric variables; ${ }^{\ddagger}$, the AIC and BIC results of the logarithmic model were better than those of the non-logarithmic model; ${ }^{\S}$, because the number of placenta percreta in the control group was 0 , the statistical model could not be calculated. *, $\mathrm{P}<0.05 ;{ }^{* \star}, \mathrm{P}<0.01$; ${ }^{\star \star \star}, \mathrm{P}<0.001$; $\mathrm{P}>0.05$, not significant. HA, hysteroscopic adhesiolysis; NICU, neonatal intensive care unit (NICU); AIP, abnormally invasive placenta.

Table 3 Multivariate analysis of postpartum blood loss within 24 hours in the study group and the control group

\begin{tabular}{|c|c|c|c|}
\hline Variable & B coefficient & Standard error & $P$ values \\
\hline IUA & 0.09368 & 0.04621 & $0.0436^{*}$ \\
\hline Caesarean section & 0.1387 & 0.04058 & $0.0007^{\star \star \star}$ \\
\hline Placenta integrity & -0.1633 & 0.04944 & $0.0011^{* *}$ \\
\hline Placenta accreta & 0.1185 & 0.07877 & 0.1337 \\
\hline Placenta increta & 0.7530 & 0.09737 & $<0.0001^{\star \star \star}$ \\
\hline Placenta percreta & 2.2072 & 0.2861 & $<0.0001^{\star \star \star}$ \\
\hline
\end{tabular}

There was no statistical significance difference in fetal gender distribution, birth weight, fetal distress, and NICU admissions after childbirth between the two groups $(\mathrm{P}>0.05)$. However, an Apgar score of 1 and 5 minutes after birth in the severe group was significantly lower than that in the control group $(\mathrm{P}<0.05)$ (Table 4).

Multivariate regression analysis was performed to evaluate the independent influence of variables possibly associated with postpartum blood loss within 24 hours. In the study group and the control group, IUA, caesarean section, placenta integrity, placenta increta, or placenta percreta was independently associated with change of postpartum blood loss within 24 hours (Table 3). Furthermore, in the mildto-moderate group and severe group, placenta increta or placenta percreta was independently associated with change of postpartum blood loss within 24 hours (Table 5).

\section{Discussion \\ Main findings}

In this matched retrospective cohort study, women with a HA history in the study group had a higher risk of cesarean section than the control group. Furthermore, the risks of placenta praevia, AIP and retained placenta, and PPH were substantially higher in women with an HA history when compared with the controls. The patients with the history of HA had worse obstetrical outcomes when compared to patients without the history of HA. The mild-to-moderate group had a lower risk of developing AIP, retained placenta, and $\mathrm{PPH}$ when compared with the severe group.

\section{Previous research}

Studies evaluating obstetric outcomes in pregnant women 
Table 4 The third trimester obstetrical outcomes of intrauterine operation in the intrauterine mild-to-moderate group and intrauterine severe group

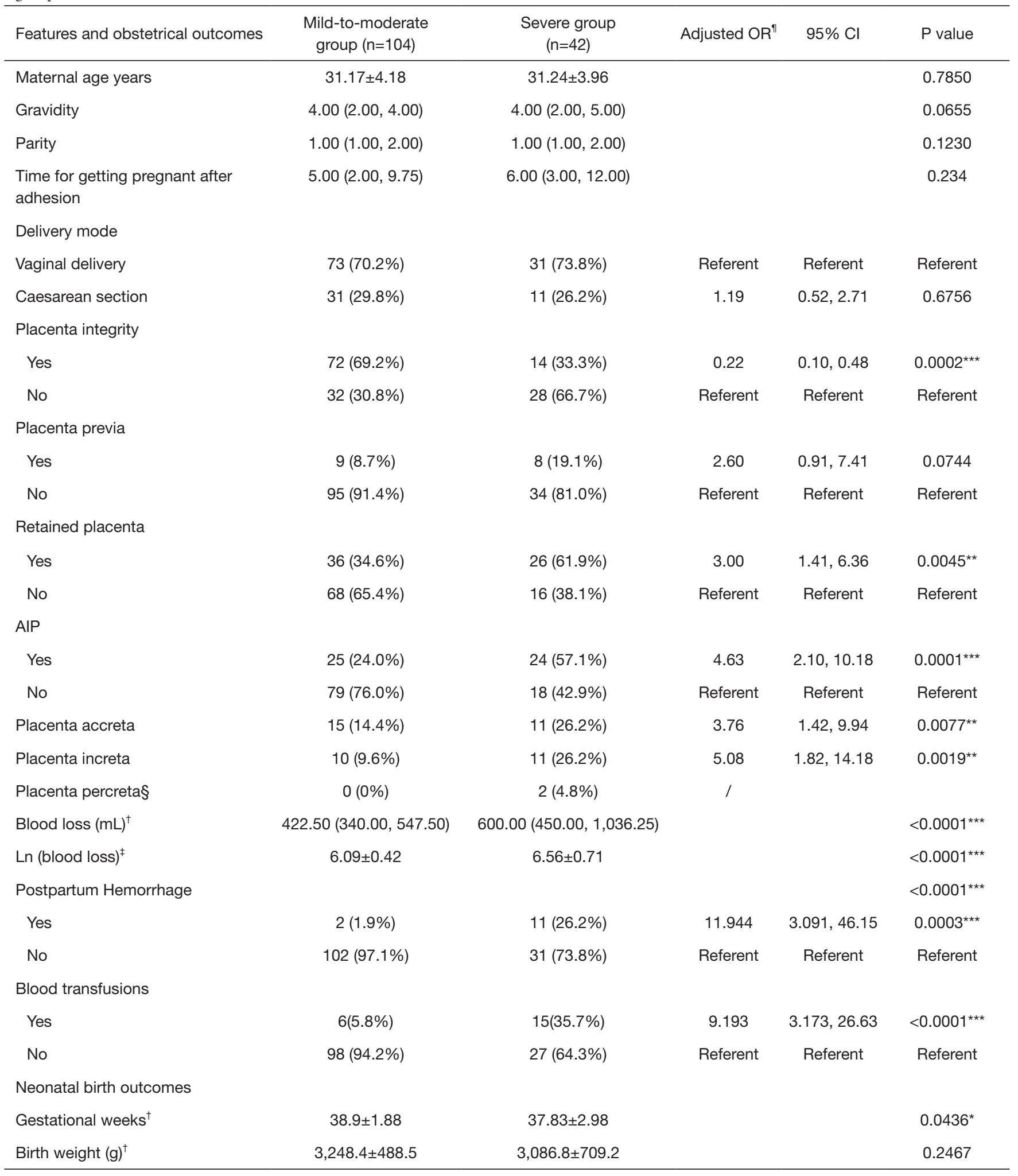

Table 4 (continued) 
Table 4 (continued)

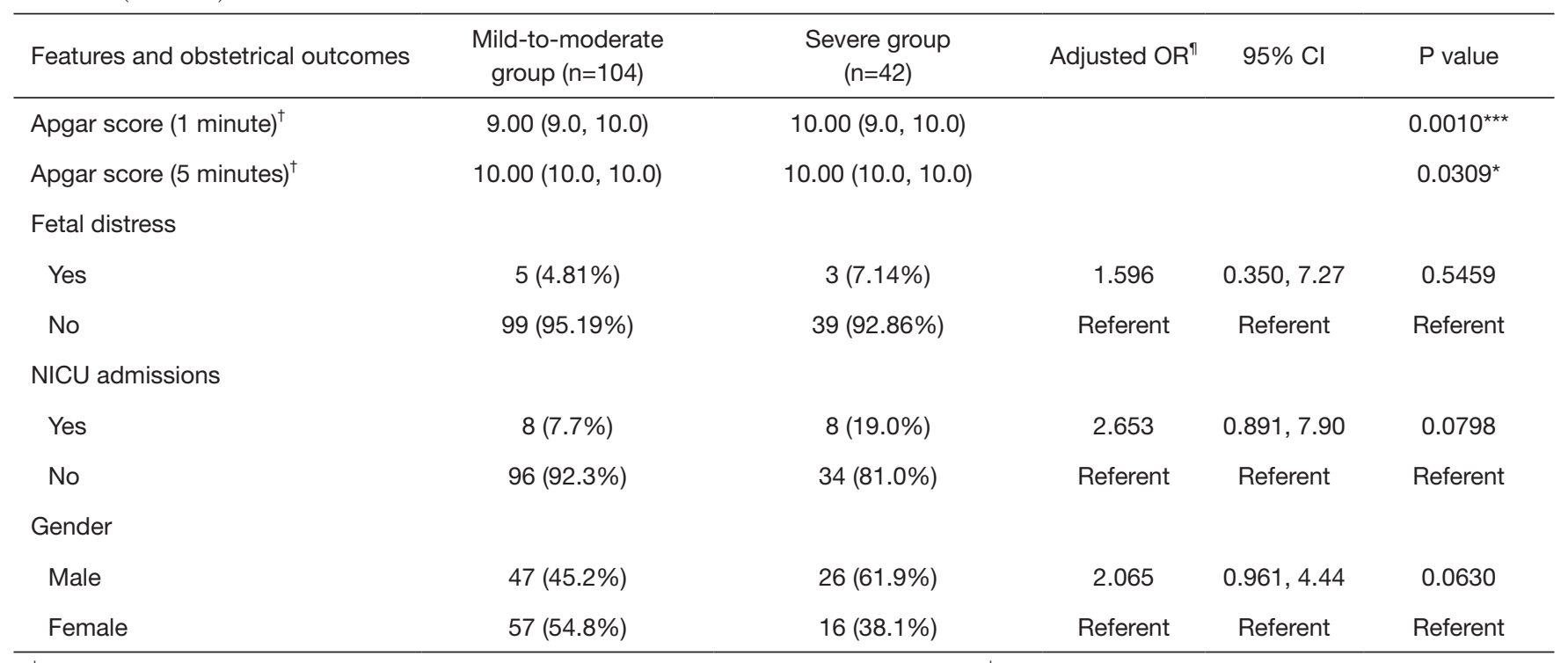

${ }^{\dagger}$, continuous variables were compared using the mixed model for parametric variables; ${ }^{\ddagger}$, the AIC and BIC results of the logarithmic model were better than those of the non-logarithmic model; ${ }^{\S}$, because the number of placenta percreta in the control group was 0 , the statistical model could not be calculated; ", the frequencies of categorical variables were maintained when adjusting for covariates of maternal age, gravidity, parity. * $\mathrm{P}<0.05$; ${ }^{\star \star}, \mathrm{P}<0.01$; ${ }^{\star \star *}, \mathrm{P}<0.001$; $\mathrm{P}>0.05$, not significant. HA, hysteroscopic adhesiolysis; NICU, neonatal intensive care unit (NICU); AIP, abnormally invasive placenta.

with HA history are sparse. Khopkar et al. reported a case of a 32-year-old woman who had morbid adhesion of placenta after hysteroscopic lysis of IUA (11). Another case report in 2015 by Engelbrechtsen $e t$ al. referred to a 38 -year-old woman who developed placenta accreta and PPH (1,000 mL) after IUA surgery. In this case report, a Bakri balloon was inserted and the bleeding subsided. There was no need for uterine sutures or hysterectomy (12). A number of references, though limited in cases, have been reported. In 1982, Schenker and Margalioth found an incidence of placenta accreta in $13-14 \%$ of patients with previous Asherman syndrome (13). Roy et al. (14) reported obstetric outcomes of 89 women following HA, of whom 32 successfully gave birth, and $4(12.5 \%)$ had PPH for adherent placenta. Chen et al. reported reproductive outcomes of 357 patients (135 with mild IUAs, 116 with moderate IUAs, and 106 with severe IUAs) who underwent HA. Of these, 17 had severe IUAs, 48 had moderate IUAs, and 75 had mild IUAs. A total of 140 cases had live births, of which 7 had PPH, including 6 cases from adherent placenta and 3 cases from placenta accreta (15).

In our present retrospective study, women with a history of HA have a higher rate of $\mathrm{PPH}(8.9 \%)$ and a higher risk
Table 5 Multivariate analysis of postpartum blood loss within 24 hours in the intrauterine mild-to-moderate group and intrauterine severe group

\begin{tabular}{lccc}
\hline Variable & B coefficient & Standard error & P values \\
\hline The severity of IUA & 0.1578 & 0.08287 & 0.0589 \\
Placenta accreta & 0.1384 & 0.08764 & 0.1165 \\
Placenta increta & 0.7414 & 0.09941 & $<0.0001^{* * *}$ \\
Placenta percreta & 2.1601 & 0.3091 & $<0.0001^{* * *}$ \\
\hline${ }^{*}, \mathrm{P}<0.05 ;$; , P<0.01; ${ }^{* * *}, \mathrm{P}<0.001 ; \mathrm{P}>0.05$, not significant. IUA, \\
intrauterine adhesion.
\end{tabular}

of AIP (33.56\%). The PPH rate and AIP rate of the mildto-moderate group were $1.9 \%$ and $57.1 \%$, corresponding to $26.2 \%$ and $24 \%$ respectively in the severe group. This may be due to the inclusion of a more severe $(n=42)$ and moderate group ( $\mathrm{n}=99)$, and the small number of mild IUA cases $(n=5)$. In addition, about $11.6 \%$ of puerperae in the study group had placenta previa, while the incidence of placenta previa of the control group was $3.1 \%$, which is consistent with the incidence of placenta previa in China (4-4.83\%) (16). It was found that pregnant women with a history of HA had a higher rate of placenta previa, and, to 
our knowledge, this has been scarcely reported in previous investigations. The higher risk of AIP and placenta previa in the study group also increased the risk of cesarean section.

When we investigated the neonatal birth outcomes, it was found that there was no significant difference in fetal gender distribution, gestational weeks, birth weight, Apgar score, fetal distress, and NICU admissions after childbirth between the study group and the control group. In the comparison of neonatal outcomes between the mild-tomoderate group and the severe group, the 1 - and 5 -minute Apgar scores for neonates of women with a history of HA was lower than those of women with no history of HA. This was mainly due to the high risks of AIP in women with a history of HA. The women with a history of HA had a higher risk of medical intervention in premature delivery, which may be the cause for lower Apgar score for neonates.

\section{Mechanisms}

The cause of IUA was mainly due to the damage of endometrium. Although IUA was separated after HA surgery, cicatrisation in the uterine wall can also occur, which is similar to severely invasive placentas that are most often localized in a previous isthmic cesarean scar. Placental tissue is by nature invasive and stimulated by hypoxia to invade deeply (scar tissue is worse for blood connections) and here it may go through the scar to seek angio-connection in a neighboring organ, usually the bladder (17). Implantation may result in a "scar pregnancy" or a "placenta percreta" either covering a uterine lower segment dehiscence or invading the scar tissue (18). It was found that of the 13 patients with $\mathrm{PPH}$ in the IUA group, 12 had various degrees of AIP, and 1 delivered without placental problems (but blood loss was 2,300 $\mathrm{mL}$ within 24 hours following delivery).

The uterus in late pregnancy or at term has at any moment a through-flow of blood that corresponds to approximately one-sixth of the pregnant woman's total blood volume, setting the scene for massive bleeding from up to 200 dilated spiral arteries into the uterine cavity if the uterus does not retract and contract as expected. We believe that the scar on the uterine wall is different from that in the position of the cesarean section, which may hinder uterine contractions and might have been another cause of PPH in the study group.

\section{Strengths and limitations}

Because patients with IUA often have a history of miscarriage, or even multiple abortions, matching studies were carried out to eliminate the impacts on the results of the study caused by age, gravidity, parity, and delivery year. Such an investigation has never been previously carried out. This study not only detailed the outcomes of pregnant women in late pregnancy but also investigated the situation of newborns, allowing us to better understand the obstetric outcomes during late pregnancy.

Our study is limited by its retrospective design and small number of pregnant patients after HA. There were plenty of patients with IUA who had post-treatment pregnancy in our hospital; however, they were not easy to followup with because some of them chose local hospitals for childbirth. We did not recruit this part of the puerperae in consideration of data integrity, thus resulting in a limited number of cases. A multicenter study of patients needs be conducted with an increased number of patients in the future in order to reduce bias.

\section{Conclusions}

In conclusions, a history of HA was associated with a higher risk of cesarean-section, placenta praevia, AIP, retained placenta, and PPH. This also increased the risk to infants. Patients with history of HA, especially with severe IUA, have worse obstetrical outcomes when compared to patients without this history. Therefore, additional medical attention may be indicated for those patients with a history of HA because of the implications of placental complications and $\mathrm{PPH}$.

\section{Acknowledgments}

Funding: This study is supported by the Natural Science Foundation of China (Grant No. 81671492). The study received approval from the institutional review board of The Third Xiangya Hospital of Central South University (March 22, 2019; number: 2019-S242). B Gao is supported by the China Scholarship Council (File number 201806370178).

\section{Footnote}

Conflicts of Interest: The authors have no conflicts of interest to declare.

Ethical Statement: The authors are accountable for all aspects of the work in ensuring that questions related to the accuracy or integrity of any part of the work are appropriately investigated and resolved. The need for ethics 
approval and consent of the present study was waived by Institutional Review Board of Third Xiangya Hospital of Central South University. Written informed consent was obtained from all patients.

\section{References}

1. Mentula M, Männistö J, Gissler M, et al. Intrauterine adhesions following an induced abortion: a nationwide cohort study. BJOG 2018;125:1424-31.

2. Su L, You L, Huang HP. Risk Factors of Intrauterine Adhesion after Hysteroscopic Resection of Endometrial Polyps. Zhongguo Yi Xue Ke Xue Yuan Xue Bao 2017;39:812-6.

3. Bhandari S, Bhave P, Ganguly I, et al. Reproductive Outcome of Patients with Asherman's Syndrome: A SAIMS Experience. J Reprod Infertil 2015;16:229-35.

4. Zhang A, Jamail G, Xue M, et al. Hysteroscopic Intrauterine Adhesiolysis Using the "Ploughing" Technique With Cold Scissors. J Minim Invasive Gynecol 2015;22:934-5.

5. Deans R, Vancaillie T, Ledger W, et al. Live birth rate and obstetric complications following the hysteroscopic management of intrauterine adhesions including Asherman syndrome. Hum Reprod 2018;33:1847-53.

6. Dilbaz B, Akpinar F, Altinbas S, et al. Reproductive Outcome of Patients With Asherman's Syndrome After Hysteroscopic Adhesiolysis. J Minim Invasive Gynecol 2015;22:S185-6.

7. Zhao J, Chen Q, Cai D, et al. Dominant factors affecting reproductive outcomes of fertility-desiring young women with intrauterine adhesions. Arch Gynecol Obstet 2017;295:923-7.

8. The American Fertility Society classifications of adnexal adhesions, distal tubal occlusion, tubal occlusion secondary to tubal ligation, tubal pregnancies, Müllerian anomalies, and intrauterine adhesions. Fertil Steril 1988;49:944-55

9. American College of Obstetricians and Gynecologists.

Cite this article as: Feng Q, Gao B, Huang $\mathrm{H}$, Woo JJ, Zou L, Zhao X, Cheng C, Xu D. Obstetrical outcome in the third trimester after hysteroscopic adhesiolysis. Ann Transl Med 2020;8(4):51. doi: 10.21037/atm.2019.09.123
reVITALize obstetric data definitions. Available online: http://www.acog.org/-/media/Departments/PatientSafety-and-Quality-Improvement/2014reVITALizeObstet ricDataDefinitionsV10.pdf. Accessed July 25, 2018.

10. Su HW, Yi YC, Tseng JJ, et al. Maternal outcome after conservative management of abnormally invasive placenta. Taiwan J Obstet Gynecol 2017;56:353-7.

11. Khopkar U, Williams RM, Selinger M. Morbid adhesion of the placenta after hysteroscopic lysis of intrauterine adhesions. Fertil Steril 2006;86:1513.e1-3.

12. Engelbrechtsen L, Langhoff-Roos J, Kjer JJ, et al. Placenta accreta: adherent placenta due to Asherman syndrome. Clin Case Rep 2015;3:175-8.

13. Schenker JG, Margalioth EJ. Intrauterine adhesions: an updated appraisal. Fertil Steril 1982;37:593-610.

14. Roy KK, Baruah J, Sharma JB, et al. Reproductive outcome following hysteroscopic adhesiolysis in patients with infertility due to Asherman's syndrome. Arch Gynecol Obstet 2010;281:355-61.

15. Chen L, Zhang H, Wang Q, et al. Reproductive outcome in patients with intrauterine adhesions following hysteroscopic adhesiolysis: Experience from the Largest Women's Hospital in China. J Minim Invasive Gynecol 2017;24:299-304.

16. Lyu B, Chen M, Liu XX. Risk factors of peripartum hysterectomy in placenta previa: a retrospective study of 3840 cases. Zhonghua Fu Chan Ke Za Zhi 2016;51:498-502.

17. Langhoff-Roos J, Chantraine F, Geirsson RT. AIP (abnormally invasive placenta) - from a retained placenta to destruction of the uterine wall. Acta Obstet Gynecol Scand 2013;92:367-8.

18. Jauniaux E, Jurkovic D. Placenta accreta: Pathogenesis of a 20th century iatrogenic uterine disease. Placenta 2012;33:244-51. 